\title{
Mortalidade por câncer bucal e de orofaringe no Brasil, de 2000 a 2013: tendências por estratos sociodemográficos
}

\author{
Mortality from oral and oropharyngeal cancer in Brazil, \\ between 2000 and 2013: trends by sociodemographic strata
}

Amanda Ramos da Cunha (https://orcid.org/0000-0002-6358-5582) ${ }^{1}$

Taiane Schaedler Prass (https://orcid.org/0000-0003-3136-909X) ${ }^{2}$

Fernando Neves Hugo (https://orcid.org/0000-0003-2222-7719) ${ }^{1}$

${ }^{1}$ Faculdade de Odontologia, Universidade Federal do Rio Grande do Sul (UFRGS) R. Ramiro Barcelos 2492, Santa Cecília. 90035-003 Porto Alegre RS Brasil. amandaracunha@ yahoo.com.br

${ }^{2}$ Instituto de Matemática e Estatística, UFRGS. Porto Alegre RS Brasil.

\begin{abstract}
Generalized observations of temporal trends in mortality could mask consistent specific patterns. This study aims to analyze the trend of oral and oropharyngeal cancer mortality rates in Brazil, from 2000 to 2013, considering the differences by gender, anatomical site, age group and ethnicity. Data on oral and oropharyngeal cancer mortality were retrieved from the Mortality Information System. The trend of historical series mortality rates by stratum was estimated through a generalized linear regression by the Prais -Winsten method. In total, 61,190 deaths from oral and oropharyngeal cancer were recorded in the 2000-2013 period (mean of coefficients: 3.50 deaths/100 thousand inhabitants/year). The trend of mortality rates was stable for males and increasing for females (1.31\%/year). A growing pattern was identified for men aged 20-29 years (2.92\%/year) and brown men (20.36\%/year). The increasing pattern was also identified for white women (2.70\%/year) and brown women (8.24\%/year). We can conclude that surveillance of this condition should consider the sociodemographic differences of the population for equitable planning of care strategies because they reflected in different trends of oral and oropharyngeal cancer mortality rates in Brazil.
\end{abstract}

Key words Mouth neoplasms, Oropharyngeal neoplasms, Mortality, Ethnicity and Health
Resumo Observações generalizadas de tendências temporais de mortalidade podem encobrir padrões específicos relevantes. O objetivo deste estudo é analisar a tendência das taxas de mortalidade por câncer bucal e de orofaringe no Brasil, no período de 2000 a 2013, considerando as diferenças por sexo, sítio anatômico, faixa etária e raça/cor. Os dados sobre a mortalidade por câncer bucal e de orofaringe foram obtidos do Sistema de Informações sobre Mortalidade. A tendência das taxas de mortalidade da série histórica, por estrato, foi estimada por regressão linear generalizada pelo método de Prais-Winsten. De 2000 a 2013, ocorreram 61.190 óbitos por essa doença (média de 3,50 óbitos/100 mil hab./ano). A tendência das taxas mostrou-se estacionária para homens e crescente para mulheres (1,31\%/ano). Identificou-se padrão de crescimento para homens de 20-29 anos (2,92\%/ano) e para homens pardos (20,36\%/ano). Padrão de crescimento também foi identificado para mulheres brancas (2,70\%/ano) epardas (8,24\%/ano). Conclui-se que a vigilância dessa condição deve considerar as diferenças sociodemográficas da população para um planejamento equânime das estratégias de cuidado, pois estas refletiram em padrões distintos de tendência das taxas mortalidade por câncer bucal e de orofaringe no Brasil.

Palavras-chave Neoplasias bucais, Neoplasias orofaringeas, Mortalidade, Origem étnica e saúde 


\section{Introdução}

O câncer bucal e de orofaringe é um tema amplamente estudado e sua relevância é corroborada por dados epidemiológicos recentes. Segundo a Agência Internacional de Pesquisa em Câncer (IARC) estima-se, para o ano de 2020, aproximadamente 370.000 novos casos de câncer bucal, 97.000 de câncer de orofaringe e 240.000 mortes por essas neoplasias no mundo ${ }^{1}$. Considerando a população masculina, o câncer bucal apresenta a $11^{\text {a }}$ e $12^{\mathrm{a}}$ maiores taxas ajustadas de incidência e mortalidade, respectivamente, entre todos os tipos de câncer; ressalta-se que estas estimativas não contabilizam as neoplasias de amígdala e de orofaringe ${ }^{2,3}$.

No Brasil, para o ano de 2018, estimou-se cerca de 15 mil novos casos de câncer bucal e de orofaringe, o que representa aproximadamente 3,5\% de todos os novos casos de câncer no país neste ano. Esse número distribui-se em um risco estimado de 10,86 casos novos a cada 100 mil homens e de 3,28 a cada 100 mil mulheres. Para o sexo masculino, é o $5^{\circ}$ câncer mais incidente no Brasil, chegando a ocupar a $4^{\text {a }}$ posição na região Sudeste ${ }^{4}$. Em relação à mortalidade, no ano de 2015 ocorreram 5.900 óbitos por essa patologia, o que resultou em uma taxa de mortalidade, ajustada por idade, de 3,05 óbitos a cada 100.000 pessoas $^{5}$.

O consumo de tabaco e de álcool é compreendido como o principal fator etiológico para o desenvolvimento do câncer bucal ${ }^{6-8}$, enquanto o Papilomavírus Humano (HPV) passou, recentemente, a ser considerado o principal fator etiológico para o câncer de orofaringe ${ }^{9-11}$. Entende-se que a tendência temporal das taxas de incidência e mortalidade por essa doença, dentre outros aspectos, tem refletido as mudanças na exposição das populações aos fatores de risco. Em alguns países desenvolvidos, como Japão, Austrália, Canadá e Estados Unidos, observa-se um padrão decrescente ou estacionário na incidência das neoplasias da cavidade bucal - as quais estão mais fortemente relacionadas à exposição ao tabaco ${ }^{12}$. Em contrapartida, a incidência das neoplasias em sítios anatômicos associados à presença do vírus HPV tem apresentado tendência crescente nestas mesmas regiões, principalmente em homens mais jovens. Especula-se a associação desse padrão com aspectos relacionados ao comportamento sexual das populações ${ }^{3,12,13}$.

A magnitude e a tendência das taxas de mortalidade por câncer bucal e de orofaringe, além de espelharem a exposição a fatores de risco, também são influenciadas por aspectos sociodemo- gráficos e socioeconômicos e pela disponibilidade, eficácia e qualidade do tratamento ofertado aos doentes ${ }^{14-16}$. Piores condições socioeconômicas, refletidas tanto por variáveis de nível individual, como escolaridade e ocupação, quanto de nível contextual, como IDH e Produto Interno Bruto (PIB), estão associadas a piores desfechos, incluindo óbito ${ }^{16}$. A disponibilidade de acesso oportuno aos serviços de saúde é também um aspecto importante para a mortalidade por essa doença, pois está relacionada à oportunidade de diagnóstico precoce. É ainda mais relevante porque o atraso no diagnóstico é uma situação sobremaneira frequente para esse tipo de câncer (mais de 50\% dos casos) e está relacionado à detecção dos tumores em estágios avançados ${ }^{17-19}$.

Diante destes aspectos, entende-se que a vigilância das taxas de mortalidade nas populações, principalmente se analisadas por estratos, pode auxiliar no planejamento e na formulação de estratégias e programas de saúde mais equânimes, tanto no âmbito da prevenção e do diagnóstico precoce, quanto do tratamento e reabilitação $0^{20}$. Recentemente, a tendência das taxas de mortalidade por câncer bucal e de faringe no Brasil, entre os anos de 2002 e 2013, foi analisada segundo sexo, sítio anatômico e macrorregião do país ${ }^{21}$. Este é um período particularmente interessante, pois abrange os anos iniciais da reestruturação da atenção pública à saúde bucal no Brasil. O presente estudo visa analisar a tendência das taxas de mortalidade por câncer bucal e de orofaringe no Brasil em uma série temporal similar (de 2000 a 2013), entretanto, considerando diferentes estratos: além de "sítio anatômico", o presente trabalho apresenta a análise por faixa etária e por raça/ cor e a variação entre os sexos nesses estratos.

\section{Metodologia}

Este trabalho é classificado como um estudo ecológico de séries temporais. Os dados sobre a mortalidade por câncer bucal e de orofaringe entre 2000 e 2013 foram obtidos através do Sistema de Informações sobre Mortalidade (SIM), gerido pelo Ministério da Saúde do Brasil e disponibilizados pelo Portal do Departamento de Informática do SUS (DATASUS). Foram incluídos os óbitos, de indivíduos com 15 anos ou mais, causados por neoplasias malignas da cavidade bucal e da orofaringe, distribuídos nas categorias de C00 a C10 da Classificação Internacional de Doenças $10^{\mathrm{a}}$ revisão $(\mathrm{CID}-10)^{22}$. Os dados referentes à população brasileira foram obtidos através do Departamento de Informática do SUS (DATASUS), 
que agrega informações provenientes dos Censos Demográficos e das Projeções Intercensitárias do país realizados pelo Instituto Brasileiro de Geografia e Estatística (IBGE).

Neste estudo, todas as taxas de mortalidade (número de óbitos por câncer de boca e de orofaringe por 100.000 habitantes) analisadas e apresentadas foram previamente padronizadas por sexo e por faixa etária (15 a 19; 20 a 29; 30 a 39; 40 a $49 ; 50$ a 59; 60 a 69; 70 a 79 anos e 80 anos e mais), pelo método direto ${ }^{23}$, utilizando como padrão a população brasileira no ano de 2010 . A padronização objetivou considerar e remover os efeitos dos fatores relativos à distribuição da população que interferem no risco do óbito por câncer bucal e de orofaringe. A média das taxas anuais representa a média aritmética das taxas de mortalidade anuais padronizadas do período de 2000 a 2013.

Os bancos de dados utilizados para a padronização apresentaram algumas limitações, que foram contornadas da seguinte maneira: 1- $\mathrm{Na}$ série histórica analisada não estão disponíveis dados por raça/cor da população brasileira nos anos censitários (2000 e 2010); para o preenchimento dessas lacunas, utilizou-se o método da progressão geométrica - para cada raça/cor, para o ano de 2000, assumiu-se $q=P 2002 / P 2001$, em que $P 2002$ é a população (de cada raça/cor) no ano de 2002 e P2001 é a população (de cada raça/ cor) no ano de 2001. Para o ano de 2010, na mesma perspectiva, assumiu-se $q=P 2009 / P 2008^{24}$. 2- Para algumas faixas etárias, de alguns anos da série histórica, o quantitativo de população indígena não está disponível, no entanto, a população das demais raças/cores e a população total estão indicadas. Neste caso, definiu-se como quantitativo de população indígena a diferença entre o total e a soma das populações das demais raças/cores. 3- Para algumas faixas etárias, de alguns anos da série histórica, a população indígena e também a amarela são desconhecidas. Neste caso, utilizouse o método da progressão geométrica para obter uma estimativa para essas duas populações - em cada ano faltante, assumiu-se $q$ como a razão da população (indígena ou amarela) dos dois anos contíguos, pertencentes à série, mais próximos da lacuna, na faixa etária em questão ${ }^{24}$. 4- Para as faixas etárias de 70 a 79 anos e de 80 anos e mais, ambos os sexos, havia muitos dados faltantes para as raças/cores indígena e amarela (para a faixa etária "80 anos e mais", é desconhecida a população indígena de todos os anos da série, por exemplo). Devido à impossibilidade de aplicação de progressão geométrica para a correção dessas lacunas, optou-se por realizar o cálculo das taxas e a análise de tendência para essas raças/cores, para ambos os sexos, excluindo estas duas faixas etárias.

Para a estimação de tendência de mortalidade da série histórica - geral e por sexo, por sítio anatômico, por faixa etária e por raça/cor -, utilizouse a regressão linear generalizada pelo método de Prais-Winsten ${ }^{25,26}$, o qual permite efetuar correção de autocorrelação de primeira ordem na análise de séries de valores organizados no tempo. Este procedimento possibilitou classificar as taxas de mortalidade como ascendentes ( $\mathrm{p}<0,05$ e coeficiente de regressão positivo), declinantes ( $\mathrm{p}<0,05$ e coeficiente de regressão negativo) ou estacionárias $(\mathrm{p}>0,05)$ e permitiu quantificar as médias anuais de aumento ou declínio dos coeficientes, de acordo com a metodologia de Antunes e Cardo$\mathrm{so}^{26}$. A verificação dos resíduos foi realizada por análise gráfica em conjunto com a análise do $\mathrm{R}^{2}$. A análise estatística foi realizada através do software “R” versão 3.2.4.

\section{Resultados}

Entre os anos de 2000 e 2013, ocorreram 61.190 óbitos por câncer bucal e de orofaringe no Brasil. A média das taxas anuais de mortalidade na série histórica foi de 3,50 óbitos a cada 100.000 habitantes para ambos os sexos, de 7,25/100.000 hab. para o sexo masculino e de 1,84/100.000 hab. para o sexo feminino - 3,94 vezes mais elevada no sexo masculino do que no feminino. A discrepância na razão entre os sexos diminuiu na comparação entre os anos inicial e final da série: no ano de 2000, a taxa de mortalidade entre os homens era 4,41 vezes maior do que entre as mulheres $(5,34$ óbitos/100.000 homens; 1,21 óbitos/100.000 mulheres); já no ano de 2013, passou a ser 3,76 vezes maior entre os homens do que entre as mulheres (5,57 óbitos/100.000 homens; 1,48 óbitos/100.000 mulheres).

Os cinco sítios anatômicos responsáveis pela maior concentração dos óbitos analisados foram "orofaringe" (31,72\%), "outras partes e de partes não especificadas da boca” (21,55\%), "outras partes e de partes não especificadas da língua" (18,95\%), "base da língua" $(8,18 \%)$ e "palato" $(4,83 \%)$. Os sítios "glândula parótida", "assoalho da boca” e "amígdala” concentraram, respectivamente, $4,51 \%, 3,66 \%$ e 3,18\% das mortes. Por fim, "outras glândulas salivares maiores e as não especificadas", "lábio" e "gengiva" foram responsáveis por $1,45 \%, 1,07 \%$ e $0,89 \%$ dos óbitos analisados, respectivamente.

A Tabela 1 apresenta a média das taxas anuais da série histórica (a cada 100.000 hab.), por 
sítio anatômico, por faixa etária e por raça/cor, considerando ambos os sexos e cada sexo separadamente. Além disso, indica a razão destas taxas entre os sexos. Em relação à localização anatômica, o sítio que apresentou a maior taxa de mortalidade foi "orofaringe", com 1,11 óbitos a cada 100.000 hab./ano, seguido de "outras partes da boca" (0,76/100.000 hab./ano). Em relação à razão entre os sexos, "base da língua" apresentou a maior discrepância, com 6,07 óbitos para homens a cada óbito para mulheres.

A análise descritiva por faixa etária demonstrou que a maior concentração de óbitos, considerando ambos os sexos, ocorreu no grupo de 50 a 59 anos - que apresentou uma média das taxas anuais de 1,02 óbitos a cada 100.000 hab. -, seguido do grupo de 60 a 69 anos $(0,87 / 100.000$ hab./ano). Em relação à análise segundo raça/ cor, a maior taxa de mortalidade, considerando ambos os sexos, foi identificada na raça/cor preta, com 3,39 óbitos a cada 100.000 hab. Na sequên- cia, encontra-se a raça/cor branca $(3,36 / 100.000$ hab./ano). Esses dados são detalhados na Tabela 1 .

$\mathrm{Na}$ análise de tendência das taxas no período estudado, por sexo, foi identificado que a taxa de mortalidade do sexo masculino passou de 5,34 a cada 100 mil hab., no ano de 2000, para 5,57 a cada 100 mil hab. no ano de 2013. Considerando a série temporal completa, essa diferença não foi estatisticamente significativa: a tendência das taxas de mortalidade para o sexo masculino apresentou-se estacionária. Para o sexo feminino, a taxa de mortalidade a cada 100 mil hab. variou de 1,21 , no ano de 2000, para 1,48 no ano de 2013. A análise estatística para esse sexo indicou uma tendência estatisticamente significativa de crescimento, com uma média de incremento anual de $1,31 \%(\mathrm{p}=0,03)$. O Gráfico 1 demonstra o padrão, global e por sexo das taxas de mortalidade no período estudado; estes resultados são detalhados na Tabela 2.

Tabela 1. Média das taxas ajustadas de mortalidade por câncer bucal e orofaringe (por 100 mil habitantes) segundo sexo, localização anatômica, faixa etária e raça/cor e razão entre as taxas (M/F). Brasil, 2000-2013.

\begin{tabular}{|c|c|c|c|c|}
\hline Variável & Global & Homens $(\mathrm{H})$ & Mulheres (M) & Razão H/M \\
\hline \multicolumn{5}{|l|}{ Localização Anatômica } \\
\hline Lábio & 0,038 & 0,058 & 0,019 & 3,05 \\
\hline Base da Língua & 0,283 & 0,498 & 0,082 & 6,07 \\
\hline Outras Partes da Língua & 0,662 & 1,080 & 0,270 & 4,00 \\
\hline Gengiva & 0,031 & 0,041 & 0,022 & 1,86 \\
\hline Assoalho da Boca & 0,126 & 0,213 & 0,046 & 4,63 \\
\hline Palato & 0,169 & 0,248 & 0,095 & 2,61 \\
\hline Outras Partes da Boca & 0,760 & 1,146 & 0,399 & 2,87 \\
\hline Glândula Parótida & 0,159 & 0,208 & 0,113 & 1,84 \\
\hline Outras Gl. Salivares Maiores & 0,051 & 0,060 & 0,042 & 1,43 \\
\hline Amígdala & 0,112 & 0,195 & 0,034 & 5,74 \\
\hline Orofaringe & 1,108 & 1,947 & 0,322 & 6,05 \\
\hline \multicolumn{5}{|l|}{ Faixa Etária } \\
\hline $15-19$ & 0,003 & 0,003 & 0,002 & 1,37 \\
\hline $20-29$ & 0,014 & 0,018 & 0,011 & 1,60 \\
\hline $30-39$ & 0,076 & 0,122 & 0,033 & 3,70 \\
\hline $40-49$ & 0,493 & 0,889 & 0,121 & 7,35 \\
\hline $50-59$ & 1,021 & 1,845 & 0,250 & 7,38 \\
\hline $60-69$ & 0,874 & 1,493 & 0,295 & 5,06 \\
\hline $70-79$ & 0,596 & 0,873 & 0,337 & 2,59 \\
\hline $80 \mathrm{e}+$ & 0,423 & 0,451 & 0,396 & 1,14 \\
\hline \multicolumn{5}{|l|}{ Raça/Cor } \\
\hline Branca & 3,355 & 5,632 & 1,221 & 4,61 \\
\hline Preta & 3,385 & 5,441 & 1,459 & 3,73 \\
\hline Parda & 2,275 & 3,565 & 1,067 & 3,34 \\
\hline Amarela $^{*}$ & 1,402 & 2,341 & 0,522 & 4,48 \\
\hline Indígena $^{*}$ & 0,883 & 1,355 & 0,442 & 3,07 \\
\hline
\end{tabular}

Fonte: Elaborada pelos autores.

*Excluídas as faixas etárias de 70 a 79 anos e de 80 anos e mais. 
As tendências por sítio anatômico são apresentadas no Gráfico 2 e os resultados detalhados na Tabela 2. Apresentaram tendência de aumento as taxas de mortalidade por neoplasia maligna da base da língua e por neoplasia maligna do assoalho da boca, com média de incremento anual de $1,5 \%$ e $2,6 \%$, respectivamente. O sítio anatômico "amígdala" foi o único com tendência decrescente, com cerca de $-2 \%$ de variação anual média.

A análise das tendências da taxa de mortalidade que considerou as faixas etárias separadamente identificou tendência de aumento para mulheres das faixas etárias " 30 a 39 anos" e " 80 anos ou mais", tendência de aumento para homens da faixa etária "20 a 29 anos" e tendência de declínio para homens das faixas etárias de "30 a 39 anos" e " 40 a 49 anos". Por fim, a análise por raça/cor revelou tendência de aumento nas taxas de mortalidade para mulheres brancas e pardas. Já para mulheres da raça/cor preta, a tendência é de declínio. Considerando o sexo masculino, tendência de aumento foi identificada também para pardos e de declínio em homens da raça/cor amarela (Tabela 3).

\section{Discussão}

O comportamento das tendências das taxas de mortalidade por câncer bucal e de orofaringe no Brasil no período de 2000 e 2013 mostrou-se bastante variado entre os estratos estudados, indicando a importância do monitoramento mais detalhado dessa condição.

A tendência das taxas globais apresentou-se estacionária para ambos os sexos e para homens e crescente para o sexo feminino. Essa tendência pode estar refletindo as variações que o país vem experimentando no padrão de consumo de tabaco. Os indicadores de tabagismo no Brasil vêm sendo acompanhados desde o ano de 1989. De 1989 a 2003, sua prevalência reduziu de $34,8 \%$ para 22,4\% (diminuição de aproximadamente $35 \%)$. Esse declínio foi observado para ambos

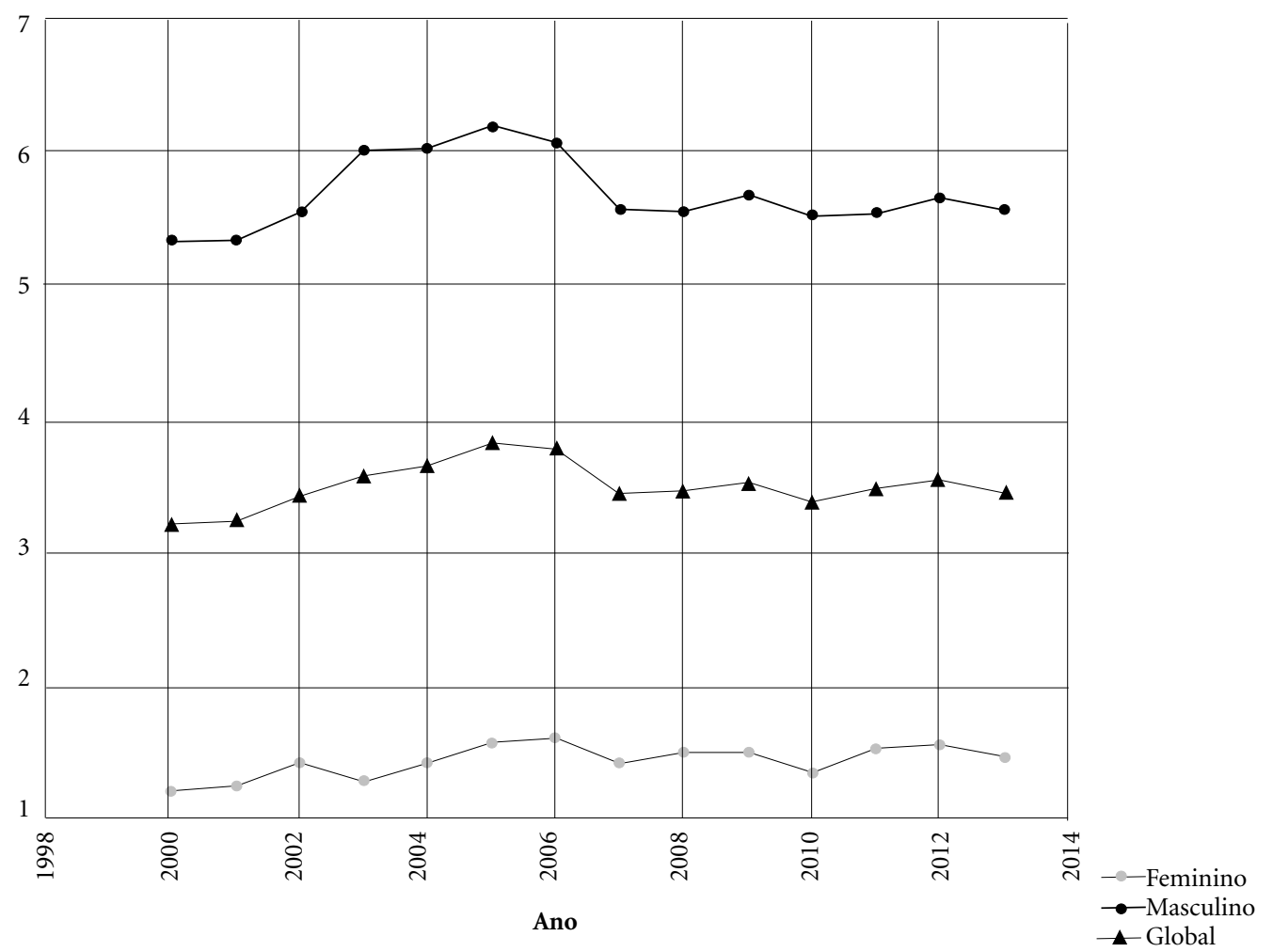

Gráfico 1. Tendência das taxas ajustadas de mortalidade por câncer bucal e de orofaringe (por 100 mil habitantes), para cada sexo e para ambos os sexos. Brasil, 2000-2013. 
Tabela 2. Tendência das taxas de mortalidade ajustadas de câncer bucal e de orofaringe, segundo localização anatômica e sexo, e porcentagem de variação anual (annual percent change - APC) das taxas. Brasil, 2000-2013.

\begin{tabular}{|c|c|c|c|c|c|c|}
\hline \multirow{2}{*}{ Variável } & \multirow[b]{2}{*}{$\operatorname{APC}(\%)$} & \multicolumn{2}{|c|}{ IC 95\% } & \multirow[b]{2}{*}{ P-valor } & \multirow[b]{2}{*}{ Tendência } & \multirow[b]{2}{*}{$\mathbf{R}^{2}$} \\
\hline & & Menor & Maior & & & \\
\hline \multicolumn{7}{|l|}{ Sítio Anatômico } \\
\hline Lábio & 0,942 & $-1,405$ & 3,345 & 0,402 & Estável & 0,997 \\
\hline Base da Língua & 1,507 & 0,398 & 2,629 & 0,012 & Crescente & 0,997 \\
\hline Outras Partes da Língua & 0,667 & $-0,447$ & 1,794 & 0,217 & Estável & 0,960 \\
\hline Gengiva & $-1,020$ & $-2,811$ & 0,804 & 0,245 & Estável & 0,999 \\
\hline Assoalho da Boca & 2,613 & 0,973 & 4,279 & 0,005 & Crescente & 0,997 \\
\hline Palato & 0,958 & $-0,092$ & 2,018 & 0,070 & Estável & 0,999 \\
\hline Outras Partes da Boca & $-0,244$ & $-1,597$ & 1,128 & 0,704 & Estável & 0,888 \\
\hline Glândula Parótida & 0,797 & $-1,599$ & 3,251 & 0,486 & Estável & 0,992 \\
\hline Outras Gl. Saliv. Maiores & $-0,624$ & $-2,690$ & 1,487 & 0,529 & Estável & 0,998 \\
\hline Amígdala & $-1,982$ & $-3,327$ & $-0,618$ & 0,008 & Decrescente & 0,998 \\
\hline Orofaringe & 0,188 & $-1,048$ & 1,440 & 0,747 & Estável & 0,551 \\
\hline \multicolumn{7}{|l|}{ Sexo } \\
\hline Mulheres & 1,307 & 0,121 & 2,507 & 0,033 & Crescente & 0,957 \\
\hline Homens & 0,175 & $-0,968$ & 1,331 & 0,746 & Estável & 0,997 \\
\hline Global & 0,413 & $-0,722$ & 1,561 & 0,445 & Estável & 0,995 \\
\hline
\end{tabular}

Fonte: Elaborada pelos autores.

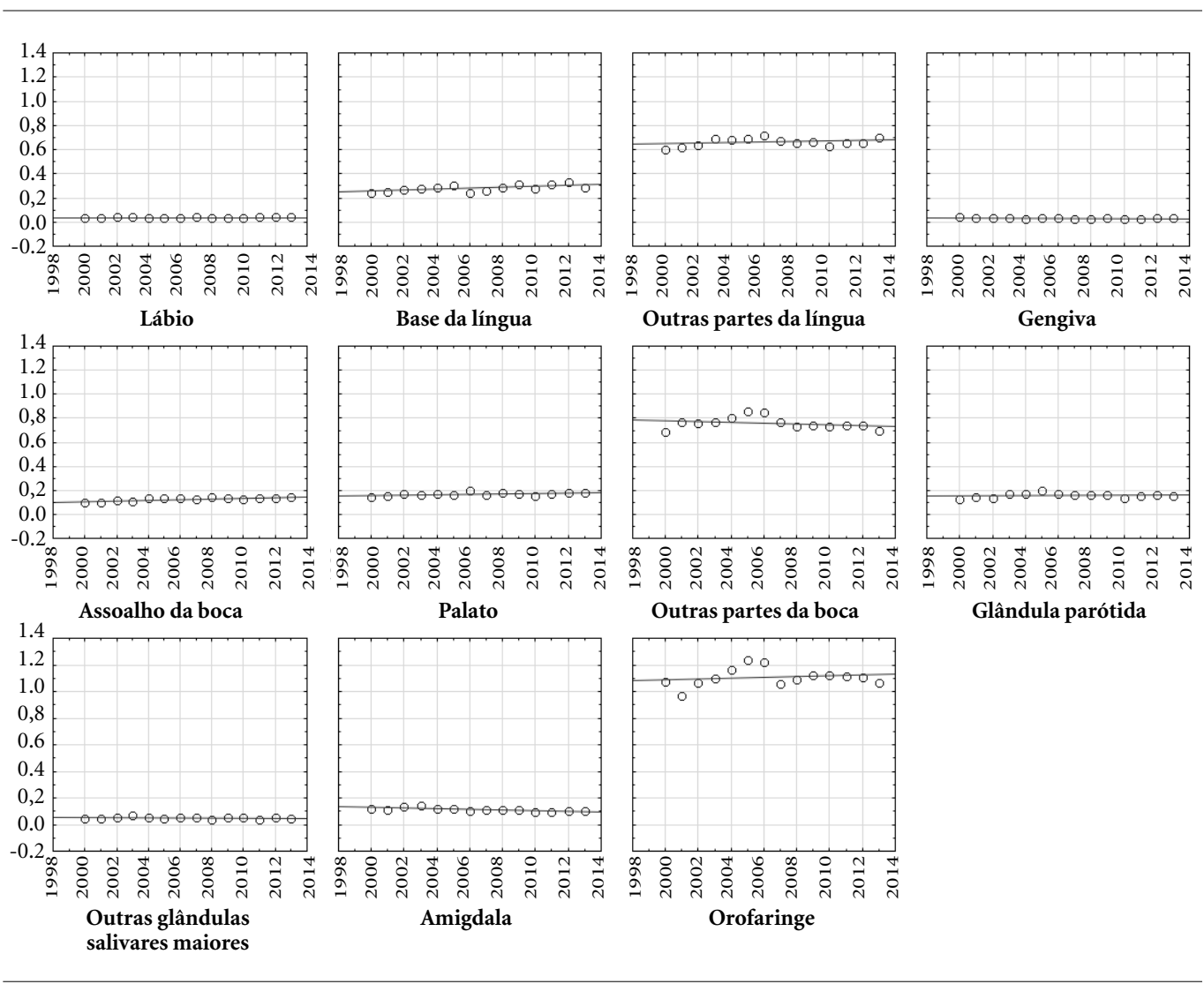

Gráfico 2. Tendência das taxas ajustadas de mortalidade por câncer bucal e de orofaringe (por 100 mil habitantes), por sítio anatômico. Brasil, 2000-2013. 
Tabela 3. Tendência das taxas de mortalidade ajustadas de câncer bucal e de orofaringe, segundo faixa etária e raça/cor, e porcentagem de variação anual (annual percent change - APC) das taxas para cada sexo. Brasil, 20002013. Fonte: Elaborada pelos autores.

\begin{tabular}{|c|c|c|c|c|c|c|}
\hline \multirow{2}{*}{ Variável } & \multirow[b]{2}{*}{$\operatorname{APC}(\%)$} & \multicolumn{2}{|c|}{ IC 95\% } & \multirow[b]{2}{*}{ P-valor } & \multirow[b]{2}{*}{ Tendência } & \multirow[b]{2}{*}{$\mathbf{R}^{2}$} \\
\hline & & Menor & Maior & & & \\
\hline \multicolumn{7}{|l|}{ Faixa Etária } \\
\hline \multicolumn{7}{|l|}{ Mulheres } \\
\hline $15-19$ & 3,733 & $-6,456$ & 15,031 & 0,455 & Estável & 0,985 \\
\hline $20-29$ & 1,298 & $-1,867$ & 4,565 & 0,394 & Estável & 0,998 \\
\hline $30-39$ & 1,961 & 0,145 & 3,810 & 0,036 & Crescente & 0,999 \\
\hline $40-49$ & 0,640 & $-1,349$ & 2,669 & 0,500 & Estável & 0,997 \\
\hline $50-59$ & 0,493 & $-0,549$ & 1,546 & 0,324 & Estável & 0,998 \\
\hline $60-69$ & 0,312 & $-0,616$ & 1,249 & 0,480 & Estável & 0,998 \\
\hline $70-79$ & 1,390 & $-0,045$ & 2,846 & 0,057 & Estável & 0,993 \\
\hline $80 \mathrm{e}+$ & 2,821 & 0,467 & 5,230 & 0,023 & Crescente & 0,971 \\
\hline \multicolumn{7}{|l|}{ Homens } \\
\hline $15-19$ & 1,805 & $-3,419$ & 7,301 & 0,474 & Estável & 0,997 \\
\hline $20-29$ & 2,915 & 0,597 & 5,286 & 0,018 & Crescente & 0,999 \\
\hline $30-39$ & $-2,598$ & $-4,047$ & $-1,128$ & 0,002 & Decrescente & 0,998 \\
\hline $40-49$ & $-1,807$ & $-3,318$ & $-0,272$ & 0,025 & Decrescente & 0,651 \\
\hline $50-59$ & 0,428 & $-0,640$ & 1,508 & 0,401 & Estável & 0,985 \\
\hline $60-69$ & 0,299 & $-0,734$ & 1,342 & 0,542 & Estável & 0,968 \\
\hline $70-79$ & 0,462 & $-0,595$ & 1,530 & 0,361 & Estável & 0,785 \\
\hline $80 \mathrm{e}+$ & 1,517 & $-0,131$ & 3,191 & 0,068 & Estável & 0,980 \\
\hline \multicolumn{7}{|l|}{ Raça/Cor } \\
\hline \multicolumn{7}{|l|}{ Mulheres } \\
\hline Amarela $^{*}$ & $-3,649$ & $-10,197$ & 3,377 & 0,272 & Estável & 0,884 \\
\hline Branca & 2,701 & 1,397 & 4,023 & 0,001 & Crescente & 0,999 \\
\hline Indígena* & $-4,339$ & $-19,211$ & 13,272 & 0,579 & Estável & 0,563 \\
\hline Parda & 8,242 & 3,481 & 13,221 & 0,002 & Crescente & 0,986 \\
\hline Preta & $-5,789$ & $-10,431$ & $-0,907$ & 0,024 & Decrescente & 0,992 \\
\hline \multicolumn{7}{|l|}{ Homens } \\
\hline Amarela $^{*}$ & $-40,386$ & $-59,298$ & $-12,688$ & 0,012 & Decrescente & 0,846 \\
\hline Branca & $-6,925$ & $-17,223$ & 4,655 & 0,207 & Estável & 0,996 \\
\hline Indígena* & $-43,994$ & $-71,635$ & 10,582 & 0,088 & Estável & 0,457 \\
\hline Parda & 20,335 & 9,782 & 31,902 & 0,001 & Crescente & 0,993 \\
\hline Preta & $-13,903$ & $-28,943$ & 4,322 & 0,115 & Estável & 0,988 \\
\hline
\end{tabular}

Excluídas as faixas etárias de 70 a 79 anos e de 80 anos e mais.

os sexos, entretanto, mostrou-se mais acentuado entre os homens do que entre as mulheres $(37 \mathrm{e}$ $32 \%$, respectivamente $)^{27}$. Padrão semelhante foi identificado na análise de recorte temporal mais recente: de 2006 a 2014, houve redução global na prevalência de tabagismo no Brasil (cerca de 0,6\% ao ano), entretanto, essa redução foi menos marcante, menos veloz e mais tardia para mulheres do que para homens ${ }^{28}$. Destaca-se que a averiguação do impacto da cessação do tabagismo na mortalidade de uma população deve contemplar o longo período de latência do desfecho, logo, o reflexo das tendências atuais do tabagismo no Brasil deve ser monitorado pelas próximas décadas.
Em relação à análise por sítio anatômico, salienta-se a tendência estável apresentada no sítio anatômico "orofaringe”, resultado compatível com estudo de série temporal semelhante ${ }^{21}$. Entre os anos de 1979 e 2002, entretanto, a mesma região anatômica apresentou taxas de mortalidade com tendência crescente no Brasil ${ }^{29}$, assim como no município de São Paulo, entre os anos 1980 e $2002^{30}$. A associação do HPV como fator etiológico de neoplasias nessa localização é comprovada na literatura ${ }^{13,31,32}$ e sabe-se que os casos associados a esse vírus têm prognóstico mais favorável $^{9,10,33}$, com pacientes apresentando menor risco de morte e de recorrência do que nos casos não 
relacionados ao $\mathrm{HPV}^{34,35}$. Essa diferença de prognóstico no câncer de orofaringe é tão relevante que a $8^{a}$ edição do Manual de Estadiamento do Câncer do American Joint Committe on Cancer (ano de 2018) passou a considerar o risco de o tumor estar associado com o HPV (identificado através de análise imuno-histoquímica) na sua categorização $^{36,37}$.

Afirmar que essa é a justificativa para a estabilidade da mortalidade nesse sítio no Brasil é precipitado, visto que o papel do HPV tem sido consistentemente identificado apenas em países economicamente desenvolvidos ${ }^{12}$, entretanto, diante da escassez de evidências que elucidem o papel do HPV no comportamento das neoplasias bucais no Brasil, essa associação não pode ser descartada. Ao encontro dessa perspectiva, estudo brasileiro de coorte identificou que pacientes com câncer de orofaringe que apresentaram resultado positivo para os anticorpos HPV16 E6/ E7 apresentaram maior sobrevida global ${ }^{38}$. Ressalta-se que, diferentemente do que é demonstrado para orofaringe, a relação do vírus HPV com o câncer bucal ainda não é bem estabelecida ${ }^{39}$.

Os resultados da análise das tendências segundo faixa etária para o sexo masculino (decrescente ou estável para todas as faixas etárias, à exceção de "20 a 29 anos") podem ser, em parte, também compreendidos pela dinâmica do tabagismo no Brasil. A prevalência de "tabagismo pesado" no país, ou seja, a que se refere aos indivíduos que fumam 20 cigarros ou mais ao dia, vem diminuindo nos últimos anos. Essa redução é mais significativa entre os homens e entre as faixas etárias de 18 a 24 anos e de 35 a $54 \operatorname{anos}^{28,40}$.

Para a faixa etária com tendência crescente, há, pelo menos, duas dimensões interpretativas: a primeira, brevemente explorada em parágrafo anterior (que aqui mantém seu caráter especulativo), diz respeito ao papel do vírus HPV na etiologia da doença, pois este parece ser mais significativo em homens e em indivíduos mais jovens ${ }^{32,41}$, o que poderia estar associado ao comportamento sexual dessa população. Recente revisão sistemática de literatura com meta-análise indicou relação entre aspectos do comportamento sexual com risco de desenvolvimento de câncer de cabeça e pescoço, com associação estatisticamente significativa entre o número de parceiros sexuais e o incremento nesse risco ${ }^{42}$. A segunda possibilidade de interpretação amparase em uma limitação estatística: o reduzido número de casos nessa faixa etária pode resultar em instabilidade na análise estatística.

Destaca-se que em relação ao sexo feminino, a faixa etária de 30 a 39 anos, que apresentou tendência de incremento, é também o estrato etário aproximado no qual há maior prevalência de consumo habitual de bebidas alcoólicas entre mulheres no Brasil ${ }^{43,44}$. O álcool configura-se como um importante fator de risco para os tumores de boca, garganta e esôfago, principalmente se associado ao tabagismo, por potencializar o efeito carcinogênico da nicotina. Considerando neoplasias de cabeça e pescoço, colorretal, de mama, de fígado e de ductos biliares, estimouse que, para mulheres brasileiras no ano de 2012, $2,6 \%$ dos casos tenham sido atribuídos ao consumo de álcool ${ }^{45}$. Ressalta-se que os dados disponíveis sobre prevalência de consumo de álcool são contemporâneos à série histórica estudada e pode-se considerar uma associação com o desfecho apenas se estes refletirem o padrão das últimas décadas.

É imperativo destacar que a mortalidade por essa patologia não está apenas relacionada à exposição aos seus fatores de risco. As taxas de sobrevivência estão associadas a fatores socioeconômicos, a oportunidades de acesso aos serviços de saúde para diagnóstico precoce e tratamento, assim como à qualidade do tratamento oferta$\mathrm{do}^{14-16}$. Na série histórica estudada, o Brasil experimentou a mais importante reestruturação dos serviços públicos de saúde bucal de sua história, com a implantação da Política Nacional de Saúde Bucal (PNSB). Essa Política reorganizou a atenção à saúde bucal, com ênfase para a ampliação do acesso através da expansão da atenção primária à saúde (APS) e para a garantia da continuidade do cuidado em nível especializado ${ }^{46}$. Estudos internacionais indicam que que estratégias para reduzir o atraso no diagnóstico do câncer bucal devem incluir a otimização dos serviços de atenção primária à saúde bucal ${ }^{47,48}$.

A PNSB apresenta as ações referentes ao câncer bucal como prioritárias. A abordagem individual inicial dessa doença é atribuição das equipes de saúde bucal da APS. É destacada, para esse nível de atenção, a importância do desenvolvimento de ações de prevenção, de detecção precoce de lesões e é encorajada atenção especial aos indivíduos com maior risco ${ }^{49}$. No período estudado, a cobertura por Equipes de Saúde Bucal da Estratégia de Saúde da Família no Brasil - equipes responsáveis pelo cuidado em saúde bucal na APS - passou de zero para cerca de $40 \%$. Diante da suspeita de lesão maligna, a equipe da APS realiza o encaminhamento do paciente para o nível secundário de atenção, representado pelos Centros de Especialidade Odontológicas (CEO), serviços responsáveis pelo diagnóstico e pelo encaminhamento para tratamento hospitalar. No período de 
2000 a 2013, o número de CEOs no Brasil passou de zero para aproximadamente $1.000^{50}$.

Todavia, pouco se sabe sobre o impacto da implementação da PNSB e da reestruturação da rede de cuidados em saúde bucal sobre o desfecho estudado. Considerando o período transcorrido, ainda insuficiente para resultados relativos à prevenção, seria o incremento de acesso aos serviços de saúde - para uma população historicamente desassistida - o principal responsável pelo reflexo na mortalidade. Essa hipótese, ainda especulativa, é fortalecida por uma característica da própria doença: o câncer bucal e de orofaringe localiza-se em uma topografia de fácil identificação à inspeção visual de um profissional capacitado, como o cirurgião-dentista, e não necessita de aparatos tecnológicos complexos para a identificação de um caso suspeito ${ }^{51}$. Neste cenário, a ampliação de acesso à APS poderia apresentar-se ainda mais potente, pois oportunizaria o diagnóstico precoce.

Sabe-se que a provisão de serviços públicos de saúde bucal no Brasil considera as desigualdades sociais, privilegiando áreas mais necessitadas ${ }^{52}$. Com a restruturação da saúde bucal no âmbito do SUS, observou-se uma redução de desigualdades no acesso e na utilização dos serviços odontológicos entre os indivíduos de maior e menor renda ${ }^{53}$. No presente estudo, a análise dos resultados do estrato raça/cor é a que mais apresenta o potencial para reflexões neste âmbito, pois as desigualdades socioeconômicas no Brasil são profundamente marcadas pelo componente étnico-racial ${ }^{54}$. A população "não branca" é identificada como desfavorecida em todos os indicadores socioeconômicos avaliados no Brasil, entre eles, anos de estudo, renda, desemprego, acesso a serviços de saúde, habitação e saneamento, etc. ${ }^{55}$. Sugere-se como hipótese, ainda especulativa, que o caráter pró-equidade da citada política pública possa estar associado com os resultados encontrados para a raça/cor preta (decrescente para mulheres e estável para homens), devido, principalmente, à ampliação de acesso aos serviços de saúde.

Para a população brasileira, há escassez de publicações que associem mortalidade por câncer bucal e raça/cor, inviabilizando comparações. Estudo que avaliou as tendências dessas taxas no município de São Paulo (de 2003 a 2009) encontrou como resultados uma tendência de incremento nas taxas de mortalidade para negros e de estabilidade para brancos ${ }^{20}$. O citado trabalho, no entanto, além de tratar de uma região específica, agregou indivíduos pretos e pardos em uma categoria, o que torna a comparabilidade pouco viável.
Os estudos internacionais recentes relacionados à mortalidade por câncer bucal e de orofaringe que consideram raça/cor são principalmente norte-americanos e têm indicado um padrão mais favorável para a população branca. As justificativas, entretanto, são direcionadas ao fato de que esta população apresenta porcentagens de neoplasias de cabeça e pescoço relacionadas ao HPV muito superiores às identificadas nas populações não brancas e esse fator etiológico está relacionado a maior sobrevida ${ }^{56,57}$.

A distribuição étnico-racial da população brasileira no território nacional é também um aspecto que deve ser considerado nas análises de tendência por raça/cor. Essa distribuição não é uniforme no Brasil; nas regiões Sul e Sudeste, predomina a população de raça/cor branca $(78$ e $55 \%$ da população, respectivamente), enquanto nas regiões Norte e Nordeste, a raça/cor preponderante é a parda (67 e 59\% da população, respectivamente $)^{58}$. A tendência das taxas para uma determinada raça/cor poderia, em parte, estar refletindo a tendência das regiões do Brasil nas quais é predominante. Estudo recente demonstrou que a única macrorregião do país que apresentou padrão de crescimento nas taxas de mortalidade por câncer bucal, no período de 2002 a 2013, foi a Nordeste ${ }^{21}$. A comparação deste resultado com o do presente estudo - que apresenta tendência de crescimento nas taxas da população parda - poderia sugerir alguma relação, a qual precisa ser investigada com a análise das taxas por raça/cor em cada macrorregião.

É preciso considerar que a mortalidade em uma série histórica reflete, em parte, as condutas terapêuticas vigentes no período e em períodos predecessores. É citado que a década de 1990 concentrou importantes avanços no tratamento dessa doença. Estudo que avaliou 3.267 pacientes tratados em uma instituição de referência no Brasil identificou que, nesta década, o tratamento cirúrgico passou a ser muito mais representativo para tumores em estágio I e II do que nas anteriores $^{59}$. Ressalta-se que, mesmo diante do achado de tendências estacionárias globais, os efeitos benéficos de avanços tecnológicos podem ser significativos, com aumento de sobrevida, aspecto não mensurado neste trabalho. Além disso, conclusões desse caráter precisam ser avaliadas também à luz da incidência da doença.

O presente trabalho apresenta limitações, que, primeiramente, relacionam-se ao seu delineamento; estudos ecológicos têm o potencial de levantar hipóteses e não são capazes de avaliar causalidade. Outro aspecto a ser discutido referese ao fato de serem analisados dados secundários, 
o que torna a validade do estudo dependente da confiabilidade das fontes. Todavia, considera-se que os dados brasileiros de óbitos por causa, ainda que apresentem um percentual de subnotificação, são tão fidedignos quanto aqueles provenientes de quaisquer outros países de longa tradição na elaboração dessas estatísticas, como Estados Unidos e outros países europeus ${ }^{60}$. Constatação semelhante é obtida quando apenas a mortalidade por câncer bucal é colocada em foco ${ }^{61}$.

A cobertura SIM no Brasil, no ano de 2011, era de $96,1 \%$. Era próxima de $100 \%$ em quase todos os estados das regiões Sudeste, Sul e Centro -Oeste, mas menor nas regióes Norte e Nordeste. $\mathrm{Na}$ região Norte, três estados apresentavam cobertura menor do que $80 \%{ }^{62}$. Essas discrepâncias não foram consideradas na análise dos dados, o que pode ter influenciado nas tendências das taxas de mortalidade principalmente da população indígena, bastante concentrada nessa região. Ademais, sublinha-se que as análises por raça/cor para indígenas e amarelos não contemplaram as faixas etárias de 70 a 79 anos e de 80 anos e mais.
Define-se como principais conclusões deste estudo que a tendência das taxas de mortalidade por câncer bucal e de orofaringe no Brasil varia significativamente quando analisada por estratos. A identificação e o monitoramento destes aspectos de maneira desagregada podem permitir uma compreensão mais fiel da dinâmica da doença estudada. A vigilância das condições de saúde em um país com características tão díspares não pode ser generalizada, ou seja, deve ater-se a características de subgrupos, para garantir a base epidemiológica para um planejamento pró-equidade; essa necessidade se aplica ao monitoramento do câncer bucal e de orofaringe no Brasil.

Ademais, conclui-se que associações da variação das tendências estudadas com a ampliação na provisão de serviços em saúde bucal no Brasil devem ser exploradas. Com base nos resultados dos estratos "sítio anatômico" e "faixa etária", o presente trabalho sugere a necessidade de estudos exploratórios referentes ao papel do HPV na etiologia do câncer bucal e de orofaringe no Brasil.

\section{Colaboradores}

AR Cunha trabalhou na concepção do projeto, na busca, análise e na interpretação dos dados, na redação do artigo e na aprovação da versão final a ser publicada. TS Prass e FN Hugo colaboraram na concepção do projeto, na análise e na interpretação dos dados, na revisão crítica relevante do conteúdo intelectual e na aprovação da versão final a ser publicada.

\section{Referências}

1. International Agency for Research on Cancer (IARC). Cancer Tomorrow [Internet]. [cited 2018 Oct 30]. Available from: http:/gco.iarc.fr/tomorrow/graphicbar?type $=1 \&$ population $=900 \&$ mode $=$ population $\&$ sex $=0 \&$ cancer $=39 \&$ age_group $=$ value $\& a p c \_$male $=$ $0 \& a p c \_$female $=0$

2. International Agency for Research on Cancer (IARC). Globocan 2012: Estimated Cancer Incidence, Mortality and Prevalence Worldwide in 2012 [Internet]. [cited 2018 Mar 8]. Available from: http://globocan.iarc.fr/ Pages/online.aspx

3. Torre LA, Bray F, Siegel RL, Ferlay J, Lortet-tieulent J, Jemal A. Global Cancer Statistics, 2012. CA a cancer J Clin 2015; 65(2):87-108.

4. Instituto Nacional de Câncer José Alencar Gomes da Silva (INCA). Estimativa 2018 - Incidência de câncer no Brasil. Rio de Janeiro: INCA; 2017.

5. Instituto Nacional de Câncer José Alencar Gomes da Silva (INCA). Atlas de Mortalidade por Câncer [Internet]. 2018 [cited 2018 Mar 8]. Available from: https:// mortalidade.inca.gov.br/MortalidadeWeb/pages/ Modelo03/consultar.xhtml\#panelResultado 
6. Huber MA, Tantiwongkosi B. Oral and Oropharyngeal Cancer. Med Clin North Am 2014; 98(6):12991321.

7. Rapidis AD, Gullane P, Langdon JD, Lefebvre JL, Scully C, Shah JP. Major advances in the knowledge and understanding of the epidemiology, aetiopathogenesis, diagnosis, management and prognosis of oral cancer. Oral Oncol 2009; 45(4-5):299-300.

8. International Agency for Research on Cancer (IARC). Personal Habits and Indoor Combustions. IARC Monogr Eval Carcinog Risks to Humans 2012; 100E:46167.

9. Chaturvedi AK. Epidemiology and Clinical Aspects of HPV in Head and Neck Cancers. Head Neck Pathol 2012; 6(1):16-24.

10. Marur S, D'Souza G, Westra WH, Forastiere AA. HPV-associated head and neck cancer: A virus-related cancer epidemic. Lancet Oncol 2010; 11(8):781-789.

11. Hobbs CGL, Sterne JAC, Bailey M, Heyderman RS, Birchall MA, Thomas SJ. Human papillomavirus and head and neck cancer: a systematic review and meta-analysis. Clin Otolaryngol 2006; 31(4):259-266.

12. Chaturvedi AK, Anderson WF, Lortet-Tieulent J, Curado MP, Ferlay J, Franceschi S, Rosenberg PS, Bray F, Gillison ML. Worldwide trends in incidence rates for oral cavity and oropharyngeal cancers. J Clin Oncol 2013; 31(36):4550-4559.

13. Chaturvedi AK, Engels EA, Anderson WF, Gillison ML. Incidence trends for human papillomavirus-related and -unrelated oral squamous cell carcinomas in the United States. J Clin Oncol 2008; 26(4):612-619.

14. Dantas TS, Silva PGB, Sousa EF, Cunha MP, Aguiar ASW, Costa FWG, Mota MRL, Alves APNN, Sousa FB. Influence of educational level, stage, and histological type on survival of oral cancer in a brazilian population: A retrospective study of 10 years observation. Medicine (Baltimore) 2016; 95(3):1-10.

15. Wünsch-Filho V. The epidemiology of oral and pharynx cancer in Brazil. Oral Oncol 2002; 38(8):737-746.

16. Boing AF, Antunes JLF. Condições socioeconômicas e câncer de cabeça e pescoço: uma revisão sistemática de literatura. Cien Saude Colet 2011; 16(2):615-622.

17. McGurk M, Chan C, Jones J, O’Regan E, Sherriff M. Delay in diagnosis and its effect on outcome in head and neck cancer. Br J Oral Maxillofac Surg 2005; 43(4):281-284.

18. Groome PA, Rohland SL, Hall SF, Irish J, Mackillop WJ, O'Sullivan B. A population-based study of factors associated with early versus late stage oral cavity cancer diagnoses. Oral Oncol 2011; 47(7):642-647.

19. Gómez I, Seoane J, Varela-Centelles P, Diz P, Takkouche $\mathrm{B}$. Is diagnostic delay related to advanced-stage oral cancer? A meta-analysis. Eur J Oral Sci 2009; 117(5):541-546.

20. Antunes JLF, Toporcov TN, Biazevic MGH, Boing AF, Bastos JL. Gender and racial inequalities in trends of oral cancer mortality in Sao Paulo, Brazil. Rev Saude Publica 2013; 47(3):470-478

21. Perea LME, Peres MA, Boing AF, Antunes JLF Tendência de mortalidade por câncer de boca e faringe no Brasil no período. Rev Saude Publica 2018; 52(10):1-10.

22. World Health Organization (WHO). ICD-10 Version: 2015 [Internet]. 2015. [cited 2018 Mar 8]. Available from: http://apps.who.int/classifications/icd10/browse /2015/en\#/C00-C14
23. Costa AJL, Kale PL, Vermelho LL. Indicadores de Saúde. In: Medronho RA, Bloch KV, Luiz RR, Werneck GL, editors. Epidemiologia. 2a . São Paulo: Atheneu; 2009. p. 31-82.

24. Von Sperling M. Wastewater Characteristics, Treatment and Disposal [Internet]. Vol. 6, Water Intelligence Online. 2007. [cited 2018 Mar 8]. Available from: http://wio.iwaponline.com/cgi/ doi/10.2166/9781780402086

25. Prais SJ, Winsten CB. Trends Estimators and Serial Correlation. Cowles Comm Discuss Pap Stat 1954; 383.

26. Antunes JLF, Cardoso MRA. Uso da análise de séries temporais em estudos epidemiológicos. Epidemiol Serv Saúde 2015; 24(3):565-576.

27. Monteiro CA, Cavalcante TM, Moura EC, Claro RM, Szwarcwald CL. Population-based evidence of a strong decline in the prevalence of smokers in Brazil (1989-2003). Bull World Health Organ 2007; 85(7):527-534

28. Malta DC, Oliveira TP, Luz M, Stopa SR, Silva Junior JB, Reis AAC. Smoking trend indicators in Brazilian capitals, 2006-2013. Cien Saude Colet 2015; 20(3):631640.

29. Boing AF, Peres MA. Mortality from oral and pharyngeal cancer in Brazil: trends and regional patterns, 1979 - 2002. Rev Panam Salud Publica 2006; 20(1):18.

30. Biazevic MGH, Castellanos RA, Antunes JLF, Michel-Crosato E. Tendências de mortalidade por câncer de boca e orofaringe no Município de São Paulo, Brasil, 1980/2002. Cad Saude Publica 2006; 22(10):21052114.

31. Califano JA, Kowalski LP, Surgery N, Hospital JH. Trends in incidence and prognosis for head and neck cancer in the United States: A site-specific analysis of the SEER database. Int J Cancer 2005; 114(5):806-816

32. Mehta V, Yu GP, Schantz SP. Population-based analysis of oral and oropharyngeal carcinoma: Changing trends of histopathologic differentiation, survival and patient demographics. Laryngoscope 2010; 120(11):2203-2212.

33. Chaturvedi AK, Engels EA, Pfeiffer RM, Hernandez BY, Xiao W, Kim E, Jiang B, Goodman MT, Sibug-Saber M, Cozen W, Liu L, Lynch CF, Wentzensen N, Jordan RC, Altekruse S, Anderson WF, Rosenberg PS, Gillison ML. Human papillomavirus and rising oropharyngeal cancer incidence in the United States. $J$ Clin Oncol 2011; 29(32):4294-4301.

34. Ragin CCR, Taioli E. Survival of squamous cell carcinoma of the head and neck in relation to human papillomavirus infection: Review and meta-analysis. Int J Cancer 2007; 121(8):1813-1820.

35. Dayyani F, Etzel CJ, Liu M, Ho CH, Lippman SM, Tsao AS. Meta-analysis of the impact of human papillomavirus (HPV) on cancer risk and overall survival in head and neck squamous cell carcinomas (HNSCC). Head Neck Oncol 2010; 2(1):1-11.

36. Lydiatt W, O'Sullivan B, Patel S. Major changes in head and neck staging for 2018. ASCO Educ B 2018; 38:505-514.

37. Lydiatt WM, Patel SG, O'Sullivan B, Brandwein MS, Ridge JA, Migliacci JC, Loomis MA, Shah JP. Head and neck cancers-major changes in the American Joint Committee on cancer eighth edition cancer staging manual. CA Cancer J Clin 2017; 67(2):122-137. 
38. López RVM, Levi JE, Eluf-Neto J, Koifman RJ, Koifman S, Curado MP, Michaluart P, Figueiredo DLA, Saggioro FP, Carvalho MB, Kowalski LP, Abrahão M, De Góis-Filho F, Tajara EH, Waterboer T, Boffetta P, Brennan P, Wünsch-Filho V. Human papillomavirus (HPV) 16 and the prognosis of head and neck cancer in a geographical region with a low prevalence of HPV infection. Cancer Causes Control 2014; 25(4):461-471.

39. Chaitanya NSK, Allam NJ, Gandhi Babu D, Waghray S, Badam R, Lavanya R. Systematic meta-analysis on association of human papilloma virus and oral cancer. J Cancer Res Ther 2016; 12(2):969.

40. Malta DC, Stopa SR, Santos MAS, Andrade SSC de A, Oliveira TP, Cristo EB, Silva MMA. Evolução de indicadores do tabagismo segundo inquéritos de telefone, 2006-2014. Cad Saude Publica 2017; 33(3):2006-2014.

41. Shiboski CH, Schmidt BL, Jordan RCK. Tongue and tonsil carcinoma: Increasing trends in the U.S. population ages 20-44 years. Cancer 2005; 103(9):18431849.

42. Farsi NJ, El-Zein M, Gaied H, Lee YCA, Hashibe M, Nicolau B, Rousseau MC. Sexual behaviours and head and neck cancer: A systematic review and meta-analysis. Cancer Epidemiol 2015; 39(6):1036-1046.

43. Moura EC, Malta DC. Alcoholic beverage consumption among adults: sociodemographic characteristics and trends. Rev Bras Epidemiol 2011; 14(3):61-70.

44. Machado ÍE, Lana FCF, Felisbino-Mendes MS, Malta DC. Factors associated with alcohol intake and alcohol abuse among women in Belo Horizonte, Minas Gerais State, Brazil | Fatores associados ao uso e abuso de álcool entre mulheres em Belo Horizonte, Minas Gerais, Brasil. Cad Saude Publica 2013; 29(7):14491459.

45. Thuler LCS, Menezes RF, Bergmann A. Cancer cases attributable to alcohol consumption in Brazil. Alcohol 2016; 54:23-26.

46. Pucca Júnior GA, Gabriel M, Araujo MED, Almeida FCS. Ten years of a national oral health policy in Brazil: Innovation, boldness, and numerous challenges. $J$ Dent Res 2015; 94(10):1333-1337.

47. Gómez I, Warnakulasuriya S, Varela-Centelles PI, López-Jornet P, Suárez-Cunqueiro M, Diz-Dios P, Seoane J. Is early diagnosis of oral cancer a feasible objective? Who is to blame for diagnostic delay? Oral Dis 2010; 16(4):333-342.

48. Dios PD, González NP, Lestón JS, Carmona IT, Posse JL, Varela-Centelles P. "Scheduling delay" in oral cancer diagnosis: A new protagonist. Oral Oncol 2005; 41(2):142-146.

49. Brasil. Ministério da Saúde (MS). Cadernos de Atenção Básica $n^{\circ} 17$ - Saúde Bucal. Série A. Normas e Manuais Técnicos. Brasília: MS; 2008.

50. Brasil. Ministério da Saúde (MS). SAGE - Sala de Apoio à Gestão Estratégica [Internet]. 2018 [cited 2018 Jan 18]. Available from: http://sage.saude.gov.br/

51. Torres-pereira CC, Angelim-dias A, Melo NS, Lemos Júnior CA, Oliveira EMF. Abordagem do câncer da boca: uma estratégia para os níveis primário e secundário de atenção em saúde. Cad Saude Publica 2012; 28(Supl.):30-39.

52. Antunes JLF, Narvai PC. Políticas de saúde bucal no Brasil e seu impacto sobre as desigualdades em saúde. Rev Saude Publica 2010; 44(2):360-365.
53. Peres KG, Peres MA, Boing AF, Bertoldi AD, Bastos JL, Barros AJD. Redução das desigualdades sociais na utilização de serviços odontológicos no Brasil entre 1998 e 2008. Rev Saude Publica 2012; 46(2):250-258.

54. Leal MC, Gama SGN, Pereira APE, Pacheco VE, Carmo CN, Santos RV. A cor da dor: iniquidades raciais na atenção pré-natal e ao parto no Brasil. Cad Saude Publica 2017; 33(1):1-17.

55. Instituto de Pesquisa Econômica Aplicada (IPEA). Retrato das Desigualdades de Gênero e Raça no Brasil [Internet]. [cited 2018 Nov 5]. Available from: http:// www.ipea.gov.br/retrato/infograficos_habitacao_saneamento.html

56. Chernock RD, Zhang Q, El-Mofty SK, Thorstad WL, Lewis JS. Human papillomavirus-related squamous cell carcinoma of the oropharynx: A comparative study in whites and African Americans. Arch Otolaryngol - Head Neck Surg 2011; 137(2):163-169.

57. Settle K, Posner MR, Schumaker LM, Tan M, Suntharalingam M, Goloubeva O, Strome SE, Haddad RI, Patel SS, Cambell EV, Sarlis N, Lorch J, Cullen KJ. Racial survival disparity in head and neck cancer results from low prevalence of human papillomavirus infection in black oropharyngeal cancer patients. Cancer Prev Res 2009; 2(9):776-781.

58. Instituto Brasileiro de Geografia e Estatística (IBGE). Sistema IBGE de Recuperação Automática - SIDRA [Internet]. [cited 2018 Nov 2]. Available from: https:// sidra.ibge.gov.br/pesquisa/censo-demografico/demografico-2010/inicial

59. Carvalho AL, Ikeda MK, Magrin J, Kowalski LP. Trends of oral and oropharyngeal cancer survival over five decades in 3267 patients treated in a single institution. Oral Oncology 2004; 40(1):71-76.

60. Laurenti R, Jorge MHPM, Gotlieb SLD. A confiabilidade dos dados de mortalidade e morbidade por doenças crônicas não-transmissíveis. Cien Saude Colet 2004; 9(4):909-920.

61. Queiroz RCS, Mattos IE, Monteiro GTR. Reliability and accuracy of oral cancer as the reported underlying cause of death in the Municipality of Rio de Janeiro. Cad Saude Publica 2003; 19(6):1645-1653.

62. Brasil. Ministério da Saúde (MS). Sistema de Informações sobre Mortalidade-SIM Consolidação da base de dados de 2011. [Internet]. [cited 2018 Nov 5]. Available from: http://tabnet.datasus.gov.br/cgi/sim/ Consolida_Sim_2011.pdf

Artigo apresentado em 16/07/2018

Aprovado em 22/11/2018

Versão final apresentada em 24/11/2018 